\title{
Antlion optimization algorithm for optimal non-smooth economic load dispatch
}

\author{
Thanh Pham Van ${ }^{1}$, Václav Snášel ${ }^{2}$, Thang Trung Nguyen ${ }^{3}$ \\ ${ }^{1}$ Faculty of Electrical Engineering and Computer Science, Technical University of Ostrava, Czech Republic \\ ${ }^{1}$ European Cooperation Center, Ton Duc Thang University, Viet Nam \\ ${ }^{2}$ Technical University of Ostrava, Czech Republic \\ ${ }^{3}$ Power System Optimization Research Group, Faculty of Electrical and Electronics Engineering, Ton Duc Thang \\ University, Viet Nam
}

\begin{tabular}{l}
\hline Article Info \\
\hline Article history: \\
Received May 26, 2019 \\
Revised Oct 5, 2019 \\
Accepted Oct 11, 2019 \\
\hline
\end{tabular}

Keywords:

Antlion optimization

Multi fuel sources

Ramp rate

Spinning reserve

Valve point loading effects

\begin{abstract}
This paper presents applications of Antlion optimization algorithm (ALO) for handling optimal economic load dispatch (OELD) problems. Electricity generation cost minimization by controlling power output of all available generating units is a major goal of the problem. ALO is a metaheuristic algorithm based on the hunting process of Antlions. The effect of ALO is investigated by solving a 10-unit system. Each studied case has different objective function and complex level of restraints. Three test cases are employed and arranged according to the complex level in which the first one only considers multi fuel sources while the second case is more complicated by taking valve point loading effects into account. And, the third case is the highest challenge to ALO since the valve effects together with ramp rate limits, prohibited operating zones and spinning reserve constraints are taken into consideration. The comparisons of the result obtained by ALO and other ones indicate the ALO algorithm is more potential than most methods on the solution, the stabilization, and the convergence velocity. Therefore, the ALO method is an effective and promising tool for systems with multi fuel sources and considering complicated constraints.
\end{abstract}

Copyright (C) 2020 Insitute of Advanced Engineeering and Science. All rights reserved.

\section{Corresponding Author:}

Thang Trung Nguyen,

Power System Optimization Research Group, Faculty of Electrical and Electronics Engineering,

Ton Duc Thang University,

19 Nguyen Huu Tho street, Tan Phong ward, District 7, Ho Chi Minh City, Viet Nam.

Email: nguyentrungthang@tdtu.edu.vn

\section{INTRODUCTION}

Minimizing electricity generation fuel cost in thermal power plants (TPPs) is extremely important because it accounts for a high rate of total electricity generation cost. So, the OELD problem has been widely applied for this purpose. So far solutions which have been just achieved by the OELD problem is to decide the power output of each thermal generating unit (TGU) so that the electricity generation fuel cost can decrease as much as possible. In addition, the OELD problem also takes many constraints into account. The constraints are power balance, spinning reserve, power output limits of generators, prohibited operating zones, and ramp rate limits. Furthermore, fuel consuming characteristics of TGU such as multi fuel sources and valve point loading effects are also considered as main issues in the OELD problem. The OELD problem has attracted many researchers because of its importance in using fuel for the TPPs reasonably. Two main method groups have used by many authors including traditional numerical methods and modern methods based on artificial intelligence.

Journal homepage: http://ijece.iaescore.com/index.php/IJECE 
Traditional numerical methods have been applied to handle the OELD problem such as Lagrangian relaxation (LR) method [1], Linear programming techniques (LPT) [2], Fast Newton raphson (FNR) method [3]. Among the methods, LR is one of the earliest methods which has been applied to systems with a quadratic fuel cost function. The constraints of the problem were rather simple such as power balance considering transmission losses, voltage limitations, and generation limits. This method was also tested on the 10-unit system and achieved results also met the requirements of technology at that time. The LPT method was combination of the Lagrangian method and linear program (LP) method in which duty of the LP method was to linearize non-linear functions and the Lagrangian method was used as usual. Therefore, when there were many nonlinear constraints, this method would face errors due to linearization. In general, all of the traditional numerical methods only have considered basic constraints of the OELD problem. Furthermore, these methods had to take the partial derivative. Consequently, traditional numerical methods will have some restrictions if they handle the OELD problem with complex constraints.

Unlike traditional numerical methods above, the modern methods have been proposed to handle the OELD problem more successfully including ANN-based methods (ANN: artificial neural network) and metaheuristic-based methods. ANN-based methods are comprised of Hopfield neural network (HNN) [4], Adaptive Hopfield neural networks (AHNN) [5], Augmented Lagrange Hopfield network (ALHN) [6] and Enhanced Augmented Lagrange Hopfield network (EALHN) [7]. Metaheuristic-based methods have been widely and more successfully handling OELD problem. Differential evolution algorithm (DEA) [8], Quantum Evolutionary Algorithm (QEA) [9], Hybrid integer coded differential evolution - dynamic programming (HICDE-DP) [10], Improved differential evolution algorithm (IDEA) [11], Colonial competitive differential evolution (CCDE) [12], Stud differential evolution (SDE) [13] and Hybrid differential evolution and Lagrange theory (HDE-LH) [14]. Real-coded genetic algorithm (RCGA) and Improved RCGA (IRCGA) [17]. Hybrid real coded genetic algorithm (HRCGA) [15], Particle swarm optimization (PSO) [16], Modified particle swarm optimization (MPSO) [18], Quantum-inspired particle swarm optimization (QIPSO) [19], Distributed sobol PSO and Tabu Search algorithm (TSA) (DSPSO-TSA) [20], Fuzzy and self adaptive particle swarm optimization (FSAPSO) [21], $\theta$-Particle swarm optimization ( $\theta$-PSO) [22], Cuckoo search algorithm (CSA) [23], [24], Improved CSA (ICSA) [25], Modified CSA (MCSA) [26], Kill herd algorithm (KHA) [27], Improved KHA (IKHA) [28], Artificial immune systems (AIS) [29], Biogeographybased optimization (BBO) [30], Chaotic firefly algorithm (CFA) [31], Grey wolf optimizer (GWO) [32], [33], Crisscross optimization (CO) [34], Exchange market algorithm (EMA) [35], ALO [36], [37], Improved firefly algorithm (IFA) [38], Whale Optimization Algorithm (WOA) [39], Crow search algorithm (CrSA) [40]. Among the DEA method and its different versions, SDE [13] is the best method. This method was created by using stud crossover operator with intent to restrict the search around low quality solutions. The most complicated conditions that this method has solved include multi fuel sources and valve point loading effects. The results show that the SDE method has provided the highest quality results compared to all other methods including the GA and TSA methods in [20] and HDE-LH in [14]; however, more complicated constraints like ramp rate, spinning reserve, and prohibited operating zones were not taken into account for challenging the method. The traditional GA method was difficult to solve OELD problem, but its variants have been applied to this problem usefully. IRCGA [17] was the strongest method in GA family. In this method, an efficient real-coded genetic algorithm (RCGA) with arithmetic-average-bound crossover and wavelet mutation was presented. The solved system by method consists of 10 TGUs with valve point loading effects, multi fuel sources, ramp rate limits, prohibited operating zones, and spinning reserve. It has proven to be the most effective when comparing to other methods including PSO, new PSO, DEA, an improved genetic algorithm (IGA). DSPSO-TSA [20] is better than several methods such as TSA, GA, PSO, and other PSO variants but it was only considering multi fuel sources and valve point loading effects but power loss constraints, prohibited operating zones as well as other complicated constraints were not consist of. The IFA method in [38] seems to be the best method since it was applied for solving systems with the most complicated constraints including both constraints considered in mentioned work and all constraints in transmission power networks. All test cases could demonstrate the outstanding performance of the method. As a new approach method, the ALO method was introduced the first time by Mirjalili in 2015 [41]. Unlike the other algorithms, ALO has two population sets are an Ant colony and an Antlion colony. According to paper [41], the ALO method has handled several mathematical functions and some engineering problems such as three classical engineering problems including three-bar truss design, cantilever beam design, and gear train design. The ALO method also has been proposed for handling the OELD problem. For example, in [36] the OELD problem has handled with simple constraints 
such as power balance and generator limits constraint, or in [37] the method has tested on small-scale power systems considering valve point effects. The ALO method has shown its potential search including several systems of the OELD problem as in [36], [37]. However, the complex level of considered systems was not large and complicated enough to decide its performance. So, in order to clarify further for the efficiency of ALO need to be more research.

In this paper, the ALO method has been applied for handling the OELD problem with the most constraints and different fuel consuming characteristics. The set of constraints is power balance, spinning reserve, power output limits of the TGU, prohibited operating zones and ramp rate limits. Fuel consuming characteristics are directly related to objective functions such as piecewise quadratic functions and non-convex piecewise function. The method has been tested on three study cases and obtained results have been compared to other methods for investigation of the ALO method.

\section{PROBLEM FORMULATION}

\subsection{Major objective of the problem}

In operation process of TPPs using fossil fuels, electricity generation cost is required to be optimized. It can be mathematically formulated by:

$$
F=\sum_{s=1}^{n} F_{s}\left(P_{s}\right)
$$

where $\mathrm{n}$ is number of TGUs; $F_{s}\left(P_{s}\right)$ is the fuel cost function of the $s^{\text {th }}$ TGU.

When the system has one fuel type, the fuel cost function of the TGU can be presented in a single quadratic form as follows:

$$
F_{s}\left(P_{s}\right)=\alpha_{s} P_{s}^{2}+\beta_{s} P_{s}+\gamma_{s}
$$

where $\alpha_{s}, \beta_{s}, \gamma_{s}$ are the cost coefficients of the $s^{\text {th }}$ TGU; and $P_{s}$ is power output of the $s^{\text {th }}$ TGU.

In the case of the multi fuel sources, the fuel cost function of each generator should be represented by a piecewise quadratic function as shown in (3). However, with the case of valve effects, the cost function is more complicated as given in (4):

$$
\begin{aligned}
& F_{s}\left(P_{s}\right)= \begin{cases}\alpha_{s 1} P_{s}^{2}+\beta_{s 1} P_{s}+\gamma_{s 1}, & \text { fuel } 1 \\
\alpha_{s 2} P_{s}^{2}+\beta_{s 2} P_{s}+\gamma_{s 2}, & \text { fuel } 2 \\
\ldots & \\
\alpha_{s m} P_{s}^{2}+\beta_{s m} P_{s}+\gamma_{s m}, & \text { fuel } \mathrm{m}\end{cases} \\
& F_{s}\left(P_{s}\right)= \begin{cases}\alpha_{s 1} P_{s}^{2}+\beta_{s 1} P_{s}+\gamma_{s 1}+\left|\delta_{s 1} \times \sin \left(\epsilon_{s 1}\left(P_{s, \text { min }}-P_{s}\right)\right)\right|, & \text { fuel } 1 \\
\alpha_{s 2} P_{s}^{2}+\beta_{s 2} P_{s}+\gamma_{s 2}+\left|\delta_{s 2} \times \sin \left(\epsilon_{s 2}\left(P_{s, m i n}-P_{s}\right)\right)\right|, & \text { fuel } 2 \\
\ldots & \\
\alpha_{s m} P_{s}^{2}+\beta_{s m} P_{s}+\gamma_{s m}+\left|\delta_{s m} \times \sin \left(\epsilon_{s m}\left(P_{s, m i n}-P_{s}\right)\right)\right|, & \text { fuel } \mathrm{m} .\end{cases}
\end{aligned}
$$

where $\alpha_{s m}, \beta_{s m}, \gamma_{s m}, \delta_{s m}, \epsilon_{s m}$ are the cost coefficients of the $s^{\text {th }}$ TGU; $m$ is number of the fuel types; and $P_{s, \text { min }}$ is the minimum power output of the $s^{\text {th }}$ TGU.

\subsection{Constraints of power system and generator}

\subsubsection{Real power balance}

The total power generation should meet the total load demand $P_{\text {demand }}$ plus transmission losses $P_{\text {loss }}$ as the following rule:

$$
\sum_{s=1}^{n} P_{s}=P_{\text {demand }}+P_{\text {loss }}
$$

where $P_{\text {loss }}$ is calculated by using Kron's formula below:

$$
P_{\text {loss }}=\sum_{s=1}^{n} \sum_{h=1}^{n} P_{s} B_{s h} P_{h}+\sum_{s=1}^{n} B_{0 s} P_{s}+B_{00}
$$

where $B_{s h}, B_{0 s}$, and $B_{00}$ are loss coefficients. 


\subsubsection{Spinning reserve constraint}

All TGUs are required that total active power reserve of them should be more than or equal to the largest generating unit. The constraint requires total active power reserve of all units $P_{r s}$ must be at least equal to the power system requirement $P_{p r}$.

$$
\sum_{s=1}^{n} P_{r s} \geq P_{p r}
$$

\subsubsection{Generating capacity constraint}

The power output of each generator must not exceed its operating limits described by the following rule:

$$
P_{s, \min } \leq P_{s} \leq P_{s, \max }, \quad \text { for } \mathrm{s}=1,2, \ldots \mathrm{n}
$$

where $P_{s, \max }$ is the highest acceptable working power of the $s^{\text {th }}$ TGU.

\subsubsection{Ramp rate constraint}

Because each TGU cannot change its power output with a high step compared to its previous generation. Thus, two major conditions are added as the following inequalities:

$$
\begin{aligned}
& P_{s}-P_{s}^{0} \leq P_{s}^{r u} \quad \text { for the case of increasing power } \\
& P_{s}^{0}-P_{s} \leq P_{s}^{r d} \quad \text { for the case of decreasing power }
\end{aligned}
$$

where $P_{s}^{0}$ is initial power from the previous operating hour of generating unit; $P_{s}^{r u}$ and $P_{s}^{r d}$ are ramp up limit and ramp down limit of the $s^{t h}$ TGU.

\subsubsection{Prohibited operating zones}

Due to engineering reason that generating units must avoid operating in several operating zones as shown in the following model:

$$
P_{s, h}^{\min } \leq P_{s} \leq P_{s, h}^{\max }
$$

where $P_{s, h}^{\min }$ and $P_{s, h}^{\max }$ are the minimum and maximum power output of the $s^{t h}$ TGU in the $h^{t h}$ prohibited operating zone.

\section{ANTLION OPTIMIZATION ALGORITHM}

Initialization: In initialization of ALO algorithm, the population of Antlions is randomly produced within the upper and lower limitations as the following model:

$$
A L O_{d}=C V^{\min }+\operatorname{rand}\left(C V^{\max }-C V^{\min }\right) \quad ; \mathrm{d}=1, \ldots, N_{\text {pop }}
$$

where $N_{p o p}$ is the population size, and $C V^{\max }$ and $C V^{\min }$ are maximum and minimum limitations of control variables.

Random walk of Ant: The movement direction of Ants does not follow any rules and it is also a random walk as described in the following model:

$$
R W_{C I}=\left[0, \sum_{C I=1}^{1}\left(2 * \alpha_{C I}-1\right), \sum_{C I=1}^{2}\left(2 * \alpha_{C I}-1\right), \sum_{C I=1}^{3}\left(2 * \alpha_{C I}-1\right), \ldots, \sum_{C I=1}^{G_{\max }}\left(2 * \alpha_{C I}-1\right)\right]
$$

where $\mathrm{CI}$ is an ordinal number of the current iteration; $G_{\max }$ is the maximum number of iterations; $\alpha_{C I}$ is considered as a moving factor; and calculated by:

$$
\alpha_{C I}= \begin{cases}1 & \text { if } \quad \alpha \geq 0.5 \\ 0 & \text { otherwise }\end{cases}
$$


The restricted space of Ant: The active radius of the $j^{\text {th }}$ Ant would be more and more decreased adaptively when the current number of iterations is increased. For mathematically modeling this behavior, the following equations are used:

$$
c_{j, C I}=\frac{C V_{j}^{\min }}{\lambda} \quad \text { and } \quad d_{j, C I}=\frac{C V_{j}^{\max }}{\lambda}
$$

where $c_{j, C I}$ and $d_{j, C I}$ are down and up limitations in the active range of the $j^{\text {th }}$ Ant at iteration CI; and is a factor $\lambda$ obtained by:

$$
\lambda=10^{\beta} \frac{C I}{G_{\max }}
$$

where $\beta$ is a constant defined based on the current iteration $\mathrm{CI}\left(\beta=2\right.$ when $\mathrm{CI}>0.1^{*} G_{\max }, \beta=3$ when CI $>$ $0.5^{*} G_{\max }, \beta=4$ when CI $>0.75^{*} G_{\max }, \beta=5$ when $\mathrm{CI}>0.9^{*} G_{\max }, \beta=6$ when $\mathrm{CI}>0.95^{*} G_{\max }$ )

Sliding Ant toward Antlion: The range of activity of Ant is affected by behavior of shooting sands of Antlion. This made Ant sliding to the bottom of the trap where the massive jaw was waiting to catch prey. To describe the assumption, the two following equations are necessary:

$$
X_{j, C I}^{\min }=A L O_{j, C I}+c_{j, C I} \quad \text { and } \quad X_{j, C I}^{\max }=A L O_{j, C I}+d_{j, C I}
$$

where $A L O_{j, C I}$ is the position of the $j^{\text {th }}$ Antlion at the $C I^{t h}$ iteration; $X_{j, C I}^{\max }$ and $X_{j, C I}^{\min }$ are corresponding to newly updated upper and lower limits of control variables included in the position of Antlions.

The movement every Ant: The movement of ants is corresponding to the determination of search zones since random walk in (13) only produces an updated step size that is not related to new solutions. The random walk position of ant can be updated by the following model:

$$
X_{j, C I}=\frac{\left(R W_{C I}-R W^{\min }\right) \times\left(X_{j, C I}^{\max }-X_{j, C I}^{\min }\right)}{R W^{\max }-R W^{\min }}+X_{j, C I}^{\min }
$$

where $R W^{\max }$ and $R W^{\text {min }}$ are the minimum and maximum values of $R W_{C I}$ respectively.

As a result, the real position of Ant is updated by using two random walks around $X_{j, C I}$ and the current best solution. The purpose is to use information exchange between two other positions. The real position is obtained by:

$$
A n t_{j, C I}=\frac{X_{j, C I}^{R W}+\text { Gbest }_{C I}^{R W}}{2}
$$

where $X_{j, C I}^{R W}$ is a new solution around $X_{j, C I}$ by using random walk; Gbest ${ }_{C I}^{R W}$ is a new solution nearby the best current solution Gbest $_{C I}$.

The phase of catching prey: In the process, the assumption is that the action of catching prey happens when Ants goes inside sand. The following equation is proposed in this regard:

$$
\text { Antlion }_{j, C I}=A n t_{j, C I} \quad \text { if } F F\left(A n t_{j, C I}\right)<F F\left(\text { Antlion }_{j, C I}\right)
$$

where $F F\left(A n t_{j, C I}\right)$ and $F F\left(\right.$ Antlion $\left._{j, C I}\right)$ are the fitness function of $A n t_{j, C I}$ and $A n t l i o n_{j, C I}$ respectively.

All steps ALO method has been summarized as Figure 1.

\section{IMPLEMENTATION OF THE ALO ALGORITHM FOR OELD PROBLEMS}

\subsection{Variables of each individual of the algorithm}

The position of each Antlion includes all control variables and is initialized within limits as the following model:

$$
\begin{aligned}
X_{d} & =C V^{\text {min }}+\operatorname{rand}\left(C V^{\max }-C V^{\min }\right) \quad ; \mathrm{d}=1, \ldots, N_{\text {pop }} \\
\text { where } C V^{\text {min }} & =\left\{P_{1, \text { min }}, \ldots, P_{n-1, \min }\right\} \quad \text { and } C V^{\max }=\left\{P_{1, \text { max }}, \ldots, P_{n-1, \max }\right\}
\end{aligned}
$$

As a result, the power output $P_{n, d}$ is obtained by equation (23) following:

$$
P_{n, d}=P_{\text {demand }}+P_{\text {loss }}-\sum_{i=1}^{n-1} P_{i, d}
$$




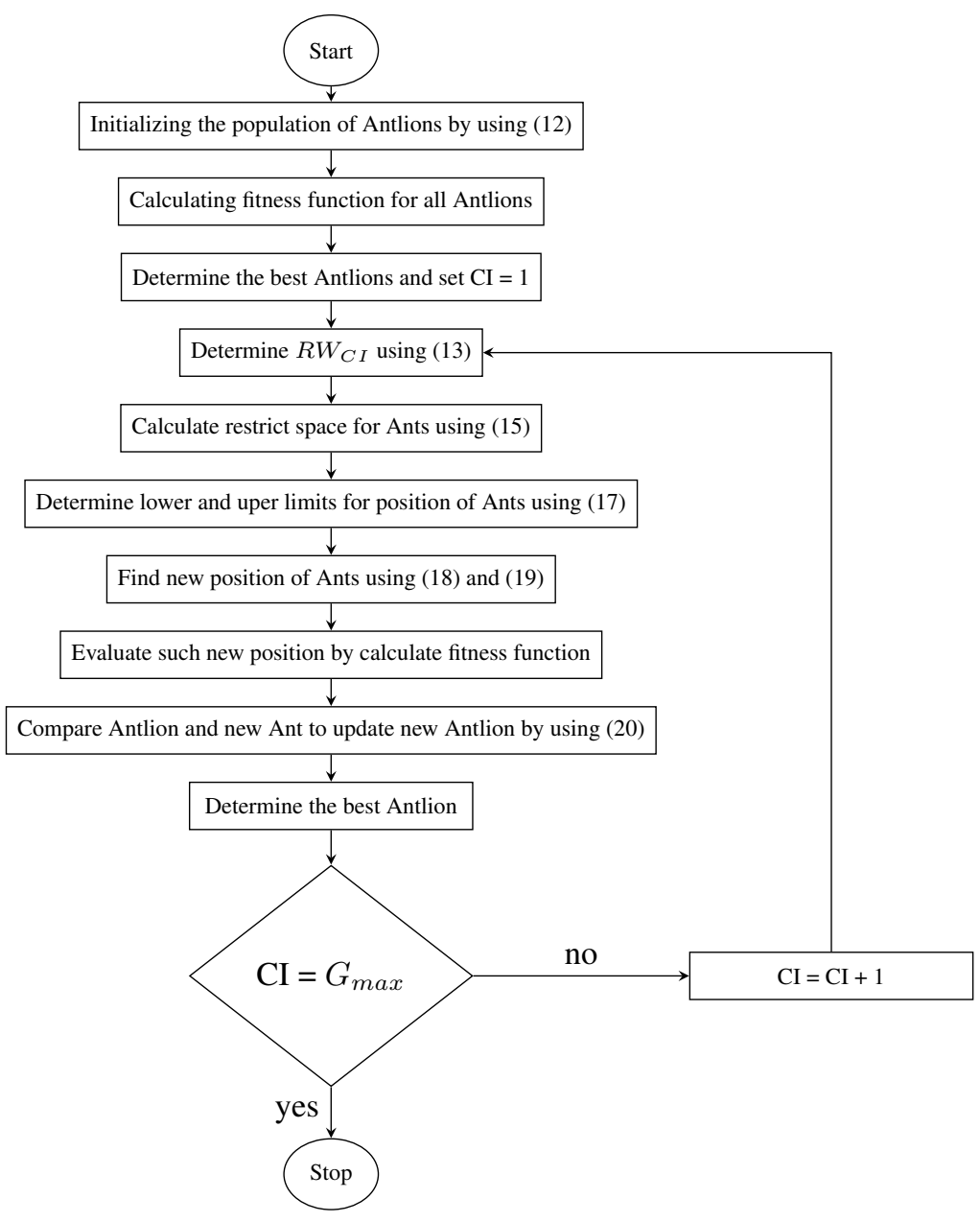

Figure 1. The flowchart of ALO algorithm

\subsection{Punishment of dependent variable violations} determined by:

In process of optimization, as $P_{n, d}$ is outside upper and lower bounds, it is penalized and

$$
\text { Cost }_{\text {Pun }}= \begin{cases}P_{n, d}-P_{n, \max } & \text { if } \quad P_{n, d}>P_{n, \max } \\ P_{n, \min }-P_{n, d} & \text { if } \quad P_{n, d}<P_{n, \min } \\ 0 & \text { if } \quad P_{n, \min } \leq P_{n, d} \leq P_{n, \max }\end{cases}
$$

\subsection{Compatible function}

The compatible function is added to the product of the square of punishment value and a punishment factor $\left(F_{a}\right)$, as following equation:

$$
C F_{d}=\sum_{i=1}^{n} F_{i}\left(P_{i}\right)+F_{a}\left(\text { Cost }_{\text {Pun }}\right)^{2}
$$

\section{NUMERICAL RESULTS}

The efficiency proposed method is judged in this section. The ALO algorithm has been tested on the 10-unit system with constraints of the power network and the generators, and different fuel consuming characteristics of thermal units. The detail is as follows:

(a) Case 1: 10-unit power system using multi fuel sources and without valve point loading effects.

(b) Case 2: 10-unit power system using multi fuel sources and valve point loading effects. 
(c) Case 3: 10-unit power system using multi fuel sources, valve point loading effects and complicated constraints such as spinning reserve, prohibited operating zones and ramp rate limits.

Data which are used for the three cases is taken from [42]. In addition, The ALO algorithm has been coded in Matlab platform and personal computer with processor $2.0 \mathrm{GHz}$ and Ram of $2.0 \mathrm{~Gb}$.

\subsection{Investigating impact of control parameters on obtained results}

Three cases above of the OELD problem have been executed by the ALO algorithm to investigate the impact of different values of control parameters in the effectiveness, robustness, and stability of the search process of ALO. Parameters have been used in the investigation are population size $\left(N_{\text {pop }}\right)$ and the number of iterations $\left(G_{\max }\right)$.

\subsubsection{Case 1: 10-unit power system using multi fuel sources and without valve point loading effects}

There are four studied subcases with four load cases from 2,400 to 2,700 MW with a change of 100 MW. The obtained results with respect to the minimum fuel cost for 100 trial runs with different cases of $N_{\text {pop }}$ and $G_{\max }$ have been reported in Table 1. Experimentation has been divided into two parts. In the first part, the population size has been kept at $N_{\text {pop }}=10$ and the number of iterations has been changed as the right first column of the Table 1. While the population size has been kept at $N_{\text {pop }}=20$ and the number of iterations as the Table 1 in the second part. Observing the table can see that the same value of $N_{\text {pop }}$, when $G_{\max }$ rises, there is a corresponding decline in the minimum fuel cost. When the minimum fuel cost equals $481.7226 \$ / h$ in both of two parts then it is impossible to decrease although $G_{\max }$ still increases. In the first part at the first six rows of Table 1 , the best costs of subcase 1.1 , subcase 1.3 and subcase 1.4 are respectively $481.7426 \$ / \mathrm{h}, 574.3808$ $\$ / \mathrm{h}, 623.8092 \$ / \mathrm{h}$ corresponding with $N_{\text {pop }}=10, G_{\max }=250$. Particularly, subcase 1.2 get the best cost at $N_{\text {pop }}=10$ and $G_{\max }=200$ with value of $526.2388 \$ / \mathrm{h}$. In the second part at the last four rows of Table 1 all four subcases reach the best cost at $G_{\max }=150$. This point out that when the population size is set to higher value, the number of iterations can be set to lower value but the best optimal solution can be found.

Table 1. The lowest cost $(\$ / \mathrm{h})$ obtained from 100 runs for different values of $N_{\text {pop }}$ and $G_{\max }$

\begin{tabular}{cccccr}
\hline $\begin{array}{c}\text { Load of } \\
2,400 \mathrm{MW}\end{array}$ & $\begin{array}{c}\text { Load of } \\
2,500 \mathrm{MW}\end{array}$ & $\begin{array}{c}\text { Load of } \\
2,600 \mathrm{MW}\end{array}$ & $\begin{array}{c}\text { Load of } \\
2,700 \mathrm{MW}\end{array}$ & $N_{\text {pop }}$ & $G_{\max }$ \\
\hline 491.2418 & 533.6331 & 579.9704 & 632.5862 & 10 & 50 \\
483.0403 & 526.5162 & 574.5738 & 624.9104 & 10 & 100 \\
481.7637 & 526.2820 & 574.3815 & 623.8695 & 10 & 150 \\
481.7424 & 526.2388 & 574.3852 & 623.8103 & 10 & 200 \\
481.7226 & 526.2388 & 574.3808 & 623.8093 & 10 & 250 \\
481.7226 & 526.2388 & 574.3808 & 623.8092 & 10 & 300 \\
483.5568 & 526.8289 & 576.4240 & 629.4534 & 20 & 50 \\
481.7424 & 526.2494 & 574.5813 & 623.8402 & 20 & 100 \\
481.7226 & 526.2388 & 574.3808 & 623.8092 & 20 & 150 \\
481.7226 & 526.2388 & 574.3808 & 623.8092 & 20 & 200 \\
\hline
\end{tabular}

The results of the distribution of the fuel costs for subcase 1.4 over 100 trials are shown in Figure 2. The results of the tests show that ALO can find the best optimal solution for different setting of control parameters and the deviation among obtained minimums is very small. Thus, ALO is stable and effective for the first case with four different loads.

\subsubsection{Case 2: 10-unit power system using multi fuel sources and valve effects}

The second study case only considers the load demand of 2,700 MW. Meantime, $N_{\text {pop }}$ has been kept at the value of 20 but $G_{\max }$ has been adjusted within 8 values from 50 to 400 with a small change of 50 . Numbers yielded from the test including minimum cost, average cost, maximum cost are presented in Table 2. As shown in Table 2, once $G_{\max }$ decrease, the fuel cost function will decrease in the first five rows. However, row 7 indicates the fuel cost function increases unintentionally although $G_{\max }$ increases equaling 300 . This is also repeated one more time at the last row. The overview on Table 2 point out that the best cost of 623.8709 $\$ / \mathrm{h}$ is obtained at $G_{\max }=350$. Meanwhile, the minimum cost at $G_{\max }=400$ is higher than $623.8709 \$ / \mathrm{h}$. Clearly, this phenomenon has been caused by the impact of valve point loading effects on the stability of the ALO search process. The most of the fuel costs for case 2 over 100 trials have distributed nearby the minimum 
value as shown in Figure 2. This shows that ALO has good stability for solving the OELD problems on the 10 -unit system with multi fuel sources and valve point loading effects.

Table 2. Result obtained by ALO for case 2 with different values of control variables

\begin{tabular}{ccccc}
\hline Lowest cost $(\$ / \mathrm{h})$ & Average cost $(\$ / \mathrm{h})$ & Highest cost $(\$ / \mathrm{h})$ & $N_{\text {pop }}$ & $G_{\max }$ \\
\hline 629.9271 & 653.2830 & 721.8110 & 20 & 50 \\
624.1303 & 638.9358 & 690.4222 & 20 & 100 \\
624.0333 & 631.6475 & 653.1102 & 20 & 150 \\
623.9216 & 628.4258 & 643.9007 & 20 & 200 \\
623.8958 & 625.8331 & 643.8601 & 20 & 250 \\
623.9309 & 626.1910 & 644.3901 & 20 & 300 \\
623.8709 & 625.6935 & 636.3510 & 20 & 350 \\
623.8878 & 625.2053 & 635.7175 & 20 & 400 \\
\hline
\end{tabular}

\subsubsection{Case 3: 10-unit power system using multi fuel sources, valve effects and complicated constraints}

In the third studied case, input parameters and obtained results are presented in Table 3. As observed from Table 3, the minimum fuel costs obtained by ALO can drop significantly from $G_{\max }=50$ to $G_{\max }=400$ and it reaches the best minimum at $G_{\max }=400$ with the cost of $624.3894 \$ / \mathrm{h}$. However, the minimum cannot be reduced since setting $G_{\max }$ to 450 and 500 , corresponding to the cost of $624.3976 \$ / \mathrm{h}$ and $624.4035 \$ / \mathrm{h}$. Clearly, the phenomenon is similar to that in case 2 but totally different from 4 subcases in case 1 . Obviously, valve point loading effects and complicated constraints have a highly significant impact on the stability of ALO. Figure 2 is shown the fuel costs after 100 trials. They are wavered between two numbers $625 \$ / \mathrm{h}$ and $630 \$ / \mathrm{h}$.

Table 3. Result obtained by ALO for case 3 with different values of control variables

\begin{tabular}{ccccc}
\hline Lowest cost $(\$ / \mathrm{h})$ & Average cost $(\$ / \mathrm{h})$ & Highest cost $(\$ / \mathrm{h})$ & $N_{\text {pop }}$ & $G_{\max }$ \\
\hline 625.0314 & 634.9521 & 658.0532 & 30 & 50 \\
624.6915 & 628.9171 & 639.8262 & 30 & 100 \\
624.5606 & 627.2422 & 637.3534 & 30 & 150 \\
624.4409 & 627.0395 & 634.9398 & 30 & 200 \\
624.4920 & 626.2745 & 632.8379 & 30 & 250 \\
624.4626 & 626.3989 & 630.5901 & 30 & 300 \\
624.4287 & 626.2675 & 630.5357 & 30 & 350 \\
624.3894 & 625.9337 & 630.5276 & 30 & 400 \\
624.3976 & 625.7483 & 630.2841 & 30 & 450 \\
624.4035 & 625.6773 & 629.0156 & 30 & 500 \\
\hline
\end{tabular}

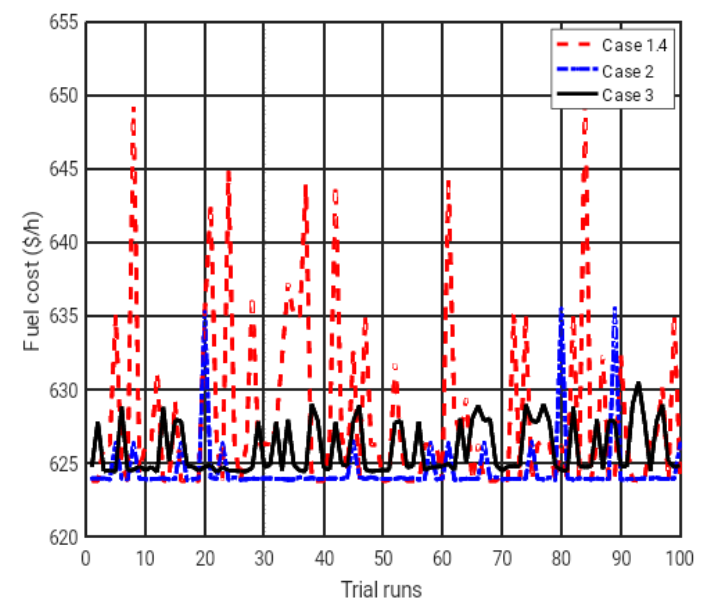

Figure 2. The best cost of 100 trials from sub-case 1.4, case 2 and case 3 


\subsection{Result comparison obtained by ALO and other methods}

In this section comparison of results obtained by the ALO method and other onces has been performed. In order to investigate the real performance of ALO method, an important factor must be concerned to be a number of fitness function evaluations $N_{f e}$, which is calculated by:

$$
N_{f e}=\omega * N_{p o p} * G_{\max }
$$

The ALO method has only one new solution generation for each iteration, so $\omega$ is 1 for ALO. For other methods such as FA and IFA in [38], $N_{f e}$ has another model, that is:

$$
N_{f e}=\frac{N_{p o p} *\left(N_{p o p}+1\right) * G_{\max }}{2}
$$

\subsubsection{Comparisons of Test Case 1}

This section compares fuel cost obtained by ALO and different methods from four subcases of case 1. The best cost together with $N_{f e}$ are reported in Table 4 . Numbers from Table 4 point out that the proposed method can harvest optimal solutions as good as others. While ALHN [6] and EALHN [7] are not metaheuristic methods. These methods will face to the difficulties of applying for OELD problem with non-convex fuel cost function and complex constraints. ALO has an approximate minimum to most methods and has better cost than FA [38] for all subcases and CFA [31] for subcase 1.4. However, as comparing $N_{f e}$ values, ALO has taken $N_{f e}=2,500$ for seeking and reaching the best optimal solutions while other methods have employed high value of $N_{f e}$, from 5,500 to 156,000. Namely, $N_{f e}$ was respectively set to 12,000, 30,000 and 156,000 for DEA, HRCGA, and CFA. In summary, ALO has found approximate or better results than compared methods but it has owned very fast convergence speed compared to other ones. In summary, the applied ALO method is really effective for case 1 with discrete objective function.

Table 4. The lowest cost $(\$ / \mathrm{h})$ of case 1 and compared methods

\begin{tabular}{llllllllllll}
\hline $\begin{array}{l}\text { Load } \\
(\mathrm{MW})\end{array}$ & $\begin{array}{l}\text { ALHN } \\
{[6]}\end{array}$ & $\begin{array}{l}\text { EALHN } \\
{[7]}\end{array}$ & $\begin{array}{l}\text { DEA } \\
{[8]}\end{array}$ & $\begin{array}{l}\text { HICDE- } \\
\text { DP } \\
{[10]}\end{array}$ & $\begin{array}{l}\text { HRCGA } \\
{[15]}\end{array}$ & $\begin{array}{l}\text { CSA } \\
{[23]}\end{array}$ & $\begin{array}{l}\text { AIS } \\
{[29]}\end{array}$ & $\begin{array}{l}\text { CFA } \\
{[31]}\end{array}$ & $\begin{array}{l}\text { FA } \\
{[38]}\end{array}$ & $\begin{array}{l}\text { IFA } \\
{[38]}\end{array}$ & ALO \\
\hline 2,400 & 481.723 & 481.723 & 481.723 & 481.7226 & 481.7226 & - & 481.723 & - & 505.2337 & 481.7226 & 481.7226 \\
2,500 & 526.239 & 526.239 & 526.239 & 526.2388 & 526.2388 & - & 526.24 & - & 580.4417 & 526.2388 & 526.2388 \\
2,600 & 574.381 & 574.381 & 574.381 & 574.3808 & 574.3808 & - & 574.381 & - & 639.287 & 574.3808 & 574.3808 \\
2,700 & 623.809 & 623.809 & 623.809 & 623.809 & 623.8092 & 623.8092 & 623.8092 & 623.8339 & 679.9525 & 623.809 & 623.8093 \\
$N_{f e}$ & - & - & 12,000 & 8,000 & 30,000 & 6,000 & 4,000 & 156,000 & 11,000 & 5,500 & 2,500 \\
\hline
\end{tabular}

\subsubsection{Comparisons of Test Case 2}

The section is carrying our comparison of results from the applied ALO and other methods for case 2. According to reported data in Table 5, KHA [27] is the best one; however, checking optimal solution reported in [27] sees that incorrect type of fuel was reported and fuel cost is much higher than reported values. Thus, KHA [27] is not a competitor of the applied ALO method. When compared to accepted methods like GA, TSA, PSO [20], FA and IFA [38], the proposed method is better with respect to the best cost. On the contrary, the ALO method is less effective than remaining methods like in DSPSO-TSA [20], CSA [23], ICSA [25]. However, it should be noted that CSA and ICSA have used $N_{f e}$ equaling 10,000 and 12,000 while that of the applied ALO method was 7,000. Compared to GA, TSA, and PSO in [20], ALO has better cost but higher $N_{f e}$ since those from ALO are 623.8708 and 7,000 while those from these methods are higher than 624.3045 and 3,000. However, results of ALO reported in Table 2 sees that ALO found the best cost of 624.1303 at $N_{\text {pop }}=$ 20 and $G_{\max }=100$, corresponding to $N_{f e}=2,000$. Thus, ALO could find better optimal solutions with faster convergence than GA, TSA, and PSO in [20]. In summary, ALO has yielded better results but its search speed has been faster than these methods while other ones with better results than ALO were slower for converging to the best optimal solution. In other words, ALO is a promising method for case 2 considering with 10 units taking multi fuel sources and valve point loading effects into account. 
Table 5. The comparison of results for case 2

\begin{tabular}{|c|c|c|c|c|c|c|c|c|c|c|c|}
\hline $\begin{array}{l}\text { Cost } \\
(\$ / h)\end{array}$ & $\begin{array}{l}\text { CCDE } \\
{[12]}\end{array}$ & $\begin{array}{l}\text { GA } \\
{[20]}\end{array}$ & $\begin{array}{l}\text { TSA } \\
{[20]}\end{array}$ & $\begin{array}{l}\text { PSO } \\
{[20]}\end{array}$ & $\begin{array}{l}\text { DSPSO- } \\
\text { TSA [20] }\end{array}$ & $\begin{array}{l}\text { CSA } \\
{[23]}\end{array}$ & $\begin{array}{l}\text { ICSA } \\
{[25]}\end{array}$ & $\begin{array}{l}\text { KHA } \\
{[27]}\end{array}$ & $\begin{array}{l}\text { FA } \\
{[38]}\end{array}$ & $\begin{array}{l}\text { IFA } \\
{[38]}\end{array}$ & ALO \\
\hline Lowest & 623.8288 & 624.505 & 624.3078 & 624.3045 & 623.8375 & 623.8684 & 623.8684 & 605.7582 & 664.5306 & 623.8768 & 623.8708 \\
\hline Average & 623.8574 & 624.7419 & 635.0623 & 624.5054 & 623.8625 & 623.9495 & 623.9495 & 605.8043 & 675.5344 & 625.2704 & 625.6935 \\
\hline$N_{f e}$ & 7,000 & 3,000 & 3,000 & 3,000 & 3,000 & 10,000 & 12,000 & 10,000 & 11,000 & 5,500 & 7,000 \\
\hline
\end{tabular}

\subsubsection{Comparisons of Test Case 3}

The section presents the comparison of fuel cost and $N_{f e}$ from the applied ALO and other methods for case 3. The results obtained for case 3 are given in Table 6. According to the results, the ALO method is only less effective than IRCGA [17] while it is better than all other methods; however, the applied ALO method has the most effective convergence speed since it has used $N_{f e}=12,000$ but that from other was much higher. For instance, the value is 33,000 for IFA [38], 66,000 for FA [38] and 90,000 for both RCGA and IRCGA. Clearly, ALO can be faster than these methods approximately from 3 times to 8 times. In summary, ALO has found a better optimal solution than three methods but less effective optimal solution than one method. However ALO is faster than all compared methods from 3 times to 8 times. Thus, ALO is a very effective method for the case, which has multi fuel sources, valve point loading effects and many complex constraints.

The optimal solution obtained by the ALO algorithm for all cases have been presented in Appendix.

Table 6. The comparison of results for case 3
\begin{tabular}{llllll}
\hline Cost & RCGA & IRCGA & FA & IFA & ALO \\
$(\$ / h)$ & {$[17]$} & {$[17]$} & {$[38]$} & {$[38]$} & \\
\hline Lowest & 624.6605 & 624.355 & 673.5544 & 624.4950 & 624.3894 \\
Average & 625.9201 & 624.5792 & 685.2872 & 625.2647 & 625.6773 \\
Highest & 628.9253 & 624.7541 & 699.2855 & 629.3951 & 629.0156 \\
$N_{f e}$ & 90,000 & 90,000 & 66,000 & 33,000 & 12,000 \\
\hline
\end{tabular}

In this section, it is explained the results of research and at the same time is given the comprehensive discussion. Results can be presented in figures, graphs, tables and others that make the reader understand easily $[2,5]$. The discussion can be made in several sub-chapters.

\section{CONCLUSION}

In this article, the proposed ALO method is effectually implemented to handle the OELD problem. The studied system has 10 TGUs with different types of fuel consuming characteristic and almost complex operation constraints of the power grid practiced in three tested cases. The method has been proved to be stable, effective and robust. The obtained results have been compared with many other methods. The comparison can imply that the ALO method is better than most other methods in term of lower fuel cost and smaller number of fitness evaluations. However, ALO has not found all better results than all methods for study cases, especially in comparison with improved versions of original meta-heuristic algorithms. Thus, it can conclude that ALO method can be selected as an optimization tool for dealing with OELD problem but it needs more improvements for enhancing optimal solution quality and converge speed.

\section{REFERENCES}

[1] Jonathan F. Bard, "Short-Term Scheduling of Thermal-Electric Generators Using Lagrangian Relaxation," IEEE Transactions on Power Systems, vol. 36(5), pp. 756-766, 1988.

[2] A. Farag, S. Al-Baiyat and T. C. Cheng, "Economic load dispatch multiobjective optimization procedures using linear programming techniques," IEEE Transactions on Power Systems, vol. 10(2), pp. 731-738, May 1995.

[3] Jiann-Fuh Chen and Shin-Der Chen, "Multiobjective power dispatch with line flow constraints using the fast Newton-Raphson method," IEEE Transactions on Energy Conversion, vol. 12(1), pp. 86-93, March 1997. 
[4] Park, J.H, Kim, Y.S, Eom, Il and Lee, K.Y, "Economic load dispatch for piecewise quadratic cost function using Hopfield neural network," IEEE Transactions on Power Systems, vol. 8(3), pp. 1030-1038, Sep 1993.

[5] K. Y. Lee, A. Sode-Yome and June Ho Park," Adaptive Hopfield neural networks for economic load dispatch," IEEE Transactions on Power Systems, vol. 13(2), pp. 519-526, May 1998.

[6] Vo Ngoc Dieu, Weerakorn Ongsakul, Jirawadee Polprasert, 'The augmented Lagrange Hopfield network for economic dispatch with multiple fuel options," Mathematical and Computer Modeling, vol. 57(1), pp. 30-39, 2013.

[7] Ngoc Vo, Dieu and Ongsakul, Weerakorn, "Economic dispatch with multiple fuel types by enhanced augmented Lagrange Hopfield network," Applied Energy, vol. 91(1), pp. 281-289, March 2012.

[8] Nasimul Noman, Hitoshi Iba, "Differential evolution for economic load dispatch problems," Electric Power Systems Research, vol. 78(8), pp. 1322-1331, Aug 2008.

[9] Aryani, Ni and Soeprijanto, Adi and Yulistya Negara, I Made and Syai'in, Mat, " Economic Dispatch using Quantum Evolutionary Algorithm in Electrical Power System involving Distributed Generators," International Journal of Electrical and Computer Engineering (IJECE), vol. 7, pp. 2365-2373, Oct 2017.

[10] R Balamurugan, S Subramanian, "Hybrid integer coded differential evolution-dynamic programming approach for economic load dispatch with multiple fuel options," Energy Conversion and Management, vol. 49(4), pp. 608-614, 2008.

[11] Coelho, Leandro and Mariani, Viviana, "Improved differential evolution algorithms for handling economic dispatch optimization with generator constraints," Energy Conversion and Management," vol. 48(5), pp. 1631-1639, May 2007.

[12] Ghasemi Mojtaba, Taghizadeh Mahdi, Ghavidel Sahand and Abbasian Abbas, "Colonial competitive differential evolution: An experimental study for optimal economic load dispatch," Applied Soft Computing, vol. 40, pp. 342-363, November 2015.

[13] Naila, et al., "Multiple Fuel Machines Power Economic Dispatch Using Stud Differential Evolution," in Energies, vol. 11(6), pp. 1393, May 2018.

[14] C. Thitithamrongchai and B. Eua-Arporn, "Hybrid Self-adaptive Differential Evolution Method with Augmented Lagrange Multiplier for Power Economic Dispatch of Units with Valve-Point Effects and Multiple Fuels, 2006 IEEE PES Power Systems Conference and Exposition, pp. 908-914, Oct 2006.

[15] Subramanian Baskar, Subbaraj P and Rao M.V.C, "Hybrid real coded genetic algorithm solution to economic dispatch problem," Computers and Electrical Engineering, vol. 29(3), pp. 407-419, May 2003.

[16] Othman, Muhammad and Salim, M.A.I. and Musirin, Professor Dr. Ismail and Salim, Nur and Othman, Mohammad Lutfi, " Dynamic economic dispatch assessment using particle swarm optimization technique," Bulletin of Electrical Engineering and Informatics , vol. 7, pp. 458-464, Sep 2018.

[17] N. Amjady and H. Nasiri-Rad, "Economic dispatch using an efficient real-coded genetic algorithm," IET Generation, Transmission Distribution, vol. 3(3), pp. 266-278, March 2009.

[18] Jong-Bae Park, Ki-Song Lee, Joong-Rin Shin and K. Y. Lee, ”A particle swarm optimization for economic dispatch with nonsmooth cost functions," IEEE Transactions on Power Systems, vol. 20(1), pp. 34-42, Feb 2005.

[19] K. Meng, H. G. Wang, Z. Dong and K. P. Wong, "Quantum-Inspired Particle Swarm Optimization for Valve-Point Economic Load Dispatch," IEEE Transactions on Power Systems, vol. 25(1), pp. 215-222, Feb 2010.

[20] S. Khamsawang, S. Jiriwibhakorn, "DSPSO-TSA for economic dispatch problem with nonsmooth and noncontinuous cost functions," Energy Conversion and Management, vol. 51(2), pp. 365-375, 2010.

[21] Taher Niknam, Hasan Doagou Mojarrad, Hamed Zeinoddini Meymand, "Non-smooth economic dispatch computation by fuzzy and self adaptive particle swarm optimization," Applied Soft Computing, vol. 11(2), pp. 2805-2817, March 2011.

[22] Vahid Hosseinnezhad, Ebrahim Babaei, 'Economic load dispatch using Theta-PSO,' International Journal of Electrical Power and Energy Systems, vol. 49, pp. 160-169, July 1013.

[23] D. N. Vo, P. Schegner and W. Ongsakul "Cuckoo search algorithm for non-convex economic dispatch," IET Generation, Transmission Distribution, vol. 7(6), pp. 645-654, June 2013.

[24] Touil, Achraf and Abdelkabir, Charkaoui and Abdellah, Boulal and Habachi, Rachid and Abdelwahed, Echchatbi, " Economic and Emission Dispatch using Cuckoo Search Algorithm," International Journal of Electrical and Computer Engineering, vol. 9, pp. 3384-3390, Oct 2019. 
[25] Thang Trung Nguyen, Dieu Ngoc Vo, "The application of one rank cuckoo search algorithm for solving economic load dispatch problems," Applied Soft Computing, vol. 37, pp. 763-773, Dec 2015.

[26] Nguyen, T.T.; Vo, D.N.; Vu Quynh, N.; Van Dai, L, "Modified Cuckoo Search Algorithm: A Novel Method to Minimize the Fuel Cost," Energies, vol. 11(6), pp. 1328, 2018.

[27] Barun Mandal, Provas Kumar Roy, Sanjoy Mandal, "Economic load dispatch using krill herd algorithm," International Journal of Electrical Power and Energy Systems, vol. 57, pp. 1-10, May 2014.

[28] Chen, G.; Lu, Z.; Zhang, Z, "Improved Krill Herd Algorithm with Novel Constraint Handling Method for Solving Optimal Power Flow Problems,” Energies, vol. 11(1), pp. 76, 2018.

[29] B.K. Panigrahi, Salik R. Yadav, Shubham Agrawal, M.K. Tiwari, "A clonal algorithm to solve economic load dispatch," Electric Power Systems Research, vol. 77(10), pp. 1381-1389, Aug 2007.

[30] A. Bhattacharya and P. K. Chattopadhyay, "Biogeography-Based Optimization for Different Economic Load Dispatch Problems," IEEE Transactions on Power Systems, vol. 25(2), pp. 1064-1077, May 2010.

[31] R. Arul, S. Velusami and G. Ravi, "Chaotic firefly algorithm to solve economic load dispatch problems," 2013 International Conference on Green Computing, Communication and Conservation of Energy (ICGCE), pp. 458-464, 2013.

[32] Kamboj, V.K., Bath, S.K. and Dhillon, J.S, "Solution of non-convex economic load dispatch problem using Grey Wolf Optimizer," Neural Computing and Applications, vol. 27(5), pp. 1301-1316, July 2016.

[33] J. Mahadevan and R. Rengaraj, "Real power dispatch with prohibited operating zones and transmission losses using grey wolf optimizer," Energy Information and Commuincation (ICCPEIC), pp. 813-823, 2017.

[34] Anbo Meng, Jinbei Li and Hao Yin, "An efficient crisscross optimization solution to large-scale nonconvex economic load dispatch with multiple fuel types and valve-point effects," Energy, vol. 113, pp. 1147-1161, Oct 2016.

[35] Naser Ghorbani and Ebrahim Babaei, "Exchange market algorithm for economic load dispatch," International Journal of Electrical Power and Energy Systems, vol. 75, pp. 19-27, Feb 2016.

[36] Menakshi Mahendru Nischal and Shivani Mehta, "Optimal Load Dispatch Using Ant Lion Optimization," Engineering Research and Applications, vol. 5(8), pp. 10-19, August 2015.

[37] Vikram Kumar Kamboj, Ashutosh Bhadoria and S. K. Bath, "Solution of non-convex economic load dispatch problem for small-scale power systems using ant lion optimizer," Neural Computing and Applications, vol. 28(8), pp. 2181-2192, August 2017.

[38] Thang Nguyen Trung, Quynh Nguyen Vu and Dai Le Van, "Improved Firefly Algorithm: A Novel Method for Optimal Operation of Thermal Generating Units," Complexity, pp. 1-23, July 2018.

[39] C k, Faseela and Vennila, H., "Economic and Emission Dispatch using Whale Optimization Algorithm," International Journal of Electrical and Computer Engineering (IJECE), vol. 8, pp. 1297-1304, June 2018.

[40] Rachid Habachi and Achraf Touil and Abdellah Boulal and Abdelkabir Charkaoui and Abdelwahed Echchatbi, " Resolution of economic dispatch problem of the morocco network using crow search algorithm," Indonesian Journal of Electrical Engineering and Computer Science, vol. 13, pp. 347-353, Jan 2019.

[41] Mirjalili Seyedali, "The Ant Lion Optimizer," Advances in Engineering Software, vol. 83, pp. 80-98, May 2015.

[42] Chao-Lung Chiang, "Improved genetic algorithm for power economic dispatch of units with valve-point effects and multiple fuels," IEEE Transactions on Power Systems, vol. 20(4), pp. 1690-1699, Nov 2005. 
Appendix

Table 7. Optimal solutions found by ALO study cases

\begin{tabular}{|c|c|c|c|c|c|c|}
\hline Power output & \multicolumn{4}{|c|}{ Case 1} & Case 2 & Case 3 \\
\hline MW & $2,400 \mathrm{MW}$ & $2,500 \mathrm{MW}$ & $2,600 \mathrm{MW}$ & $2,700 \mathrm{MW}$ & $2,700 \mathrm{MW}$ & 2,700 MW \\
\hline$P_{1}$ & 189.7408 & 206.5197 & 216.5486 & 218.2607 & 219.1333 & 218.9026 \\
\hline$P_{2}$ & 202.3362 & 206.4566 & 210.9095 & 211.7224 & 212.4026 & 213.4446 \\
\hline$P_{3}$ & 253.8923 & 265.7344 & 278.5384 & 280.7647 & 279.6938 & 284.6929 \\
\hline$P_{4}$ & 233.0488 & 235.9536 & 239.0974 & 239.6537 & 239.9551 & 240.1769 \\
\hline$P_{5}$ & 241.8215 & 258.0099 & 275.4831 & 278.3093 & 279.1825 & 259.9421 \\
\hline$P_{6}$ & 233.0420 & 235.9511 & 239.1002 & 239.6635 & 238.9891 & 241.1175 \\
\hline$P_{7}$ & 253.2723 & 268.8587 & 285.7104 & 288.7401 & 292.4503 & 294.7045 \\
\hline$P_{8}$ & 233.0428 & 235.9521 & 239.1087 & 239.5935 & 239.0145 & 240.8488 \\
\hline$P_{9}$ & 320.3769 & 331.4902 & 343.5002 & 428.3186 & 426.7170 & 436.3226 \\
\hline$P_{10}$ & 239.4264 & 255.0738 & 272.0035 & 274.9736 & 272.4617 & 269.8475 \\
\hline Cost $(\$ / h)$ & 481.7226 & 526.2388 & 574.3808 & 623.8093 & 623.8709 & 624.3894 \\
\hline
\end{tabular}

\section{BIOGRAPHIES OF AUTHORS}

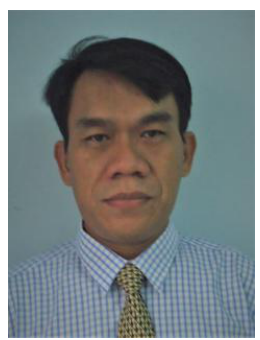

Thanh Pham received his B.Eng degrees in Electrical \& Electronics Engineering and M.Eng degrees in Automatic Control Engineering from Ho Chi Minh City University of Technology, National Ho Chi Minh University, Viet Nam in 1997 and 2004 respectively. Now he is a lecturer at faculty of electrical engineering, Cao Thang Technical College, VN. He is also a doctoral student at Technical University of Ostrava, Czech Republic. His research interests include optimization algorithms, power system operation, and Renewable Energy.

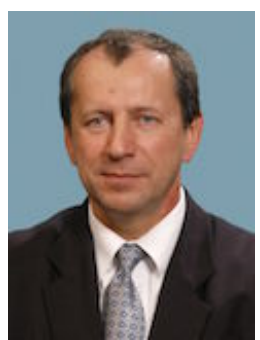

Vaclav Snasel studied numerical mathematics at Palacky University in Olomouc, and Ph.D. degree obtained at Masaryk University in Brno. Currently, he is a rector of VSB-Technical University of Ostrava. His research interests include neural network, nature and biologically inspired computing, data mining, and applied to various real-world problems.

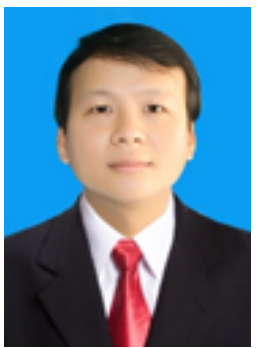

Thang Nguyen finished his PhD degree in 2018 at Ho Chi Minh City University of Technology and Education, Viet Nam. Currently, he is working at Power system optimization research group of faculty of electrical and electronics engineering. His research is about applications of metaheuristics for optimization problems in electrical engineering. 